\title{
SR-AODV: A Stable Route-AODV Protocol for Mobile Ad hoc Network
}

\author{
Pratima Gupta \\ Madan Mohan Malviya Engineering College, \\ Gorakhpur
}

\begin{abstract}
Mobile ad-hoc network is the collection of node which dynamically forms a temporary network without the use of any existing network infrastructure or centralized administration. When the mobility in the network is high and the topology changes frequently the route maintenance overhead in AODV increases. Because nodes can move link breakages can occurs. Long path is more vulnerable to link breakages and requires high control overhead for its maintenance. In this paper we introduce a new modified AODV protocol. This new protocol uses stability factor of intermediate nodes to find the most sable neighbors and therefore most stable path. This new proposed protocol is SRAODV (Stable Route AODV) protocol. We simulate both AODV and SR-AODV with ns-2 in similar scenario. We find that SR-AODV will show better performance than AODV.
\end{abstract}

Keywords AODV, Stable Route AODV, Stability Factor, Link Breakage

\section{INTRODUCTION}

The movement of the nodes affects the interconnected path of the mobile Ad-Hoc network, which in turns affect the performance of the networks. We have studied the impact of the node density of the SR-AODV \& AODV. The number of the possible connection between any two nodes is very less in sparsely populated network. So, the performance of the overall network is being poor. It is expected in the SR-AODV, when node density is increased the throughput of the network is also being increased. But after the certain level if density of the node is increased, the performance of the network decreases in some protocol. For the reliable path, selection of optimal path is one strategy through which data packets can be transmitted. There are many paths which are based on shortest path, stable path and signal quality based routes. The main challenge of these MANETs routing protocol is to provide a stable and reliable communication platform against the face of widely changing and highly mobile network. During the past time, researcher have given number of protocols AODV, DSR, DSDV, TORA are emerging routing protocols of IETF MANETs working group [1] [2] [3]. In performance comparison between conventional AODV and modified AODV protocol, modified AODV shows good performance in case of packet delivery fraction and average end to end delay, which are the most important metrics.

In this paper, we have modify the conventional AODV and proposed SR-AODV (Stable Route-AODV) protocol which is based on the concept of "path stability". In this work, our main goal is to modify the conventional AODV protocol so that selection of routing path would be stable. Selection of more stable path, minimize the number of link/path breakages and SR-AODV performers better in highly mobile network.

\section{RELATED WORK}

Many researchers have given significant contribution to the modification of AODV protocol in $\mathrm{Ad}$ hoc wireless

\author{
Dr. Rakesh Kumar \\ Madan Mohan Malviya Engineering College, \\ Gorakhpur
}

communication. They have modified conventional AODV routing protocol to increase the performance of Ad hoc networks. AODV has been extensively investigated and a number of optimizations / modifications have been suggested:

Modified/Optimized AODV Protocols

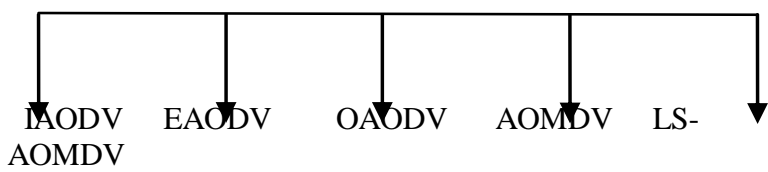

Fig.2.1: Modifications/Optimizations to AODV Protocols

AODV protocol can be implemented and evaluated for different mobility models like Random Waypoint (RWP), Reference Point Group mobility (RPGM) and Gauss Markov models (GMM) [4] and it can be shown that AODV protocol with RPGM model has optimized results for varying network load and speed. In AODV, each node has certain lifetime. If it expires, rebroadcasting of RREQ is done. In [5], Z. Jianwu et al. proposed improved AODV routing protocol (IAODV) which controls the rebroadcasting of RREQ by measuring node lifetime when sending RREQ information. Thus IAODV controlled the sending of packet more efficiently which results in better packet delivery ratio and routing load parameter. In [6], authors have proposed an enhanced AODV protocol (EAODV). In this scheme, mobile nodes are made more aware of the local connectivity to their neighbors. This is done by extending the original HELLO message in AODV but with lower overhead. Moreover, it can prevent the potential unidirectional links in the network which caused unnecessary loss of data packets. Due to node's mobility and limited transmission range, the routes created by original AODV become invalid frequently which results in large control overhead. Hence, in [7] authors have suggested optimized AODV (OAODV) based on the concept of reliable distance. This reliable distance ( $r$ ) which is always less than transmission range (R) depends on node's velocity and direction which are obtained from GPS. $r$ decides whether the node can receive RREQ from its neighbor. Thus region of flooding RREQ is restricted. By using this scheme the routes are made more reliable. A multi-path, loop free extension to AODV has been proposed in [8], which is called ad-hoc on demand multi-path distance vector routing (AOMDV). It ensures that alternate paths at every node are disjoint. Based on AOMDV, a multi-path routing protocol for real-time video transport which is called link stability-aware AOMDV (LSAOMDV) has been proposed in [9].

\section{AODV PROTOCOL}

Ad-hoc On-Demand Distance Vector Routing Protocol (AODV) that uses a demand-driven route establishment procedure. The Ad-hoc On-Demand Distance Vector outing Protocol (AODV) [3], is one of more common routing protocol in ad hoc networks and is based on the principle of discover routes as needed. AODV is a reactive protocol that has some capabilities such as; low processing, memory overhead, low network utilization. AODV routing algorithm 
builds a single loop-free path to each other node on the network. One disadvantage of AODV and most on-demand routing protocols is a route reply message loss. In route discovery phase, a route reply message (RREP) [4] of AODV obtains by the spending cost of flooding the entire network or a partial area.

\section{SR-AODV PROTOCOL}

We present a modified version of this algorithm namely Stable Route AODV (SR-AODV) protocol. The SR-AODV routing protocol can present good performance for high mobility environments. We also present our routing protocol namely Stable Route AODV (SR-AODV) routing protocol which it can be an extension for AODV protocol. SR-AODV routing protocol increases performance and when route fails occurs, the source node should select the best route between available routes. We apply stability estimation method for route selection and to increase performance.

\section{Selection of the more stable route to the destination:}

Route stability factor

$$
\begin{gathered}
n-1 \\
S_{j}= \\
i=0
\end{gathered}
$$

$$
\begin{aligned}
& \operatorname{Avg}_{\mathrm{j}} \quad=\mathrm{S}_{\mathrm{j}} / \text { Hopcount }_{\mathrm{j}} \\
& \mathrm{S}_{\mathrm{j}} \quad=\text { sum of neighbor reliability } \\
& \text { factors in the } \mathrm{j}^{\text {th }} \text { path. } \\
& \operatorname{Avg}_{j} \quad=\text { average of the summation in } \\
& \text { the } \mathrm{j}^{\text {th }} \text { path. }
\end{aligned}
$$

\section{Chosen path $=\arg \max \left\{\mathrm{A}_{\mathrm{j}}\right\}$}

Destination chooses path with highest average value. The highest average value shows the more reliable path between the source and destination. The highest average value shows the more reliable path between the source and destination. Selection of the most reliable path, the RREP packet like AODV, is broadcasted back on the selected path to the source. After receiving the RREP packet by the source node, it begins to transmit the data on this most stable path.

\section{Data transmission on selected route}

Selection of the most stable path, the RREP packets like AODV is broadcasted back on the selected path to the source. When the source node receives RREP packets, it has information about stability of available routes to destination node. So it can select a route with highest stability. When a route established between source and destination, data transmission stage can be started.

Next section comprises simulation and result analysis of the proposed modified SR-AODV protocol.

\subsection{ALGORITHM}

Behavior of an intermediate node in SR-AODV

\begin{tabular}{ll|} 
& Procedure FindRoute $(\mathrm{Ni}, \mathrm{Nj})$ \\
$* /$ & $\mathrm{S}=\mathrm{Null} ; / * \mathrm{~S}$ is the set of nodes traversed at a stage
\end{tabular}

93 | P a g e

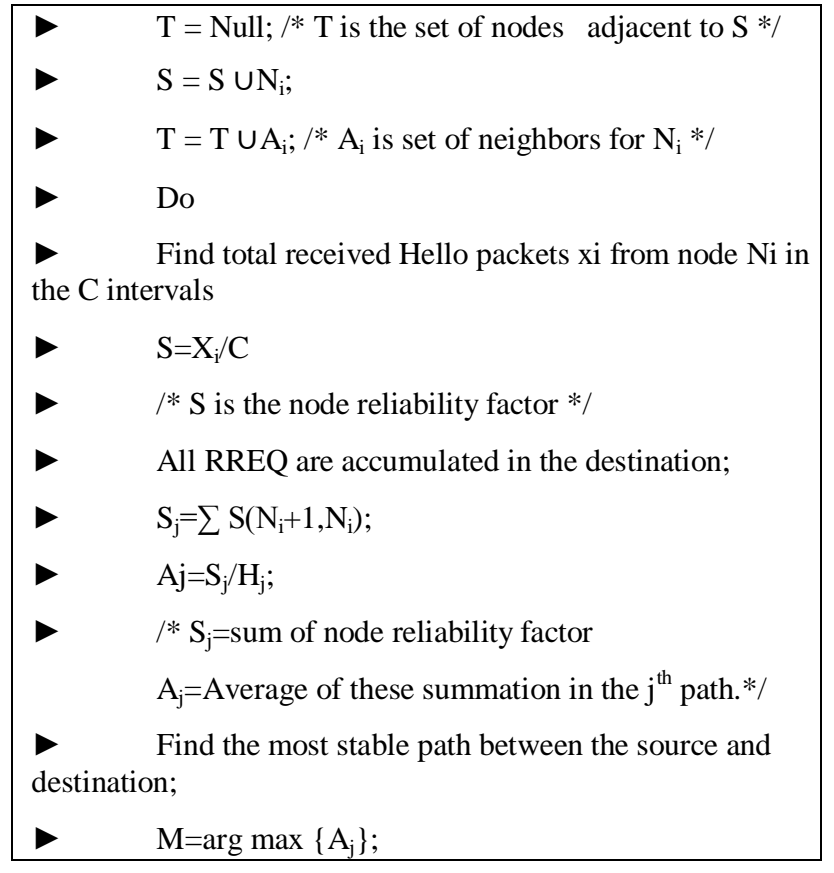

Each intermediate node which receives the RREQ message, calculates route stability factor and the route stability factor for each node is calculated by the given above equation. SRAODV algorithm solves this problem with selecting the route with maximum average value in available set of routes that have been already calculated. SR-AODV routing algorithm increases performance and when route fails occurs, the source node should select the best route between available routes.

\section{EXPERIMENTAL SETUP}

The experimental setup is used for performance evaluation of the conventional AODV \& SR-AODV routing protocols. Simulations [10] are run by considering conventional AODV $\&$ SR-AODV routing protocol. We used the ns-2 simulator to implement our routing protocol [11] for comparison with SRAODV and AODV routing protocol and to check the effectiveness of the proposed method.

Simulation parameters for packet delivery fraction \& throughput are as given below in Table 5.1:

Table 5.1: Simulation Parameters PDF

\begin{tabular}{|l|l|}
\hline \multicolumn{1}{|c|}{ Parameters } & \multicolumn{1}{c|}{ Value } \\
\hline Simulator & NS-2.29 \\
\hline Study Protocols & 150 seconds \\
\hline Simulation Time & $1000 \mathrm{~m}$ X $1000 \mathrm{~m}$ \\
\hline Simulation Area & $10 \mathrm{~m} / \mathrm{sec}$ \\
\hline Max speed & CBR \\
\hline Traffic Source & 512 byte \\
\hline Packet Size & $20,50,80,100$ \\
\hline No. of nodes &
\end{tabular}

Table 5.2: Simulation Parameters for Average End to End Delay 


\begin{tabular}{|l|l|}
\hline \multicolumn{1}{|c|}{ Parameters } & \multicolumn{1}{c|}{ Value } \\
\hline Simulator & NS-2.29 \\
\hline Study Protocols & AODV \& SR-AODV \\
\hline Simulation Time & 600 seconds \\
\hline Simulation Area & $1000 \mathrm{~m} \mathrm{X} 1000 \mathrm{~m}$ \\
\hline Max speed & $10 \mathrm{~m} / \mathrm{sec}$ \\
\hline Traffic Source & CBR \\
\hline Packet Size & 512 byte \\
\hline No. of nodes & $20,50,80,100$ \\
\hline
\end{tabular}

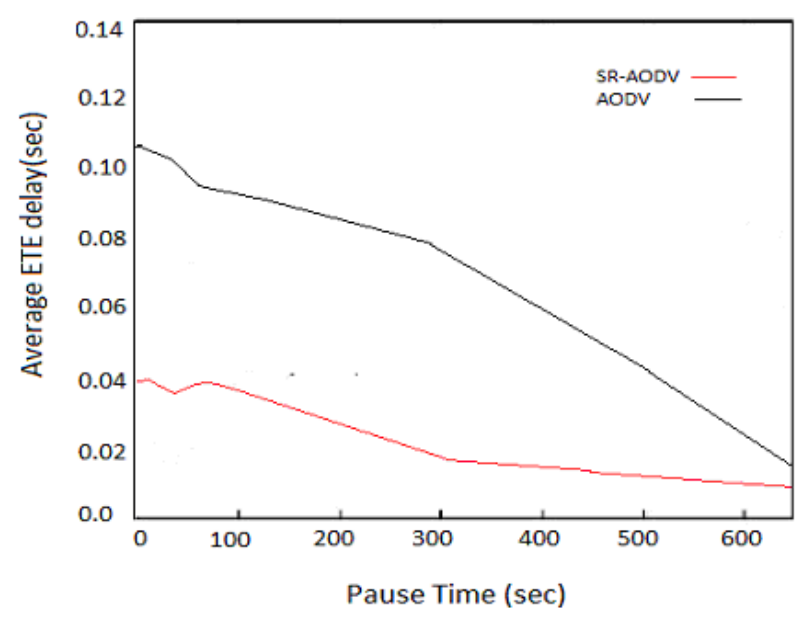

\section{PERFORMANCE METRICS \& RESULT ANALYSIS}

For evaluation of SR-AODV routing protocol performance, we used two metrics:

\section{Packet Delivery Fraction \\ 2. Average ETE Delay}

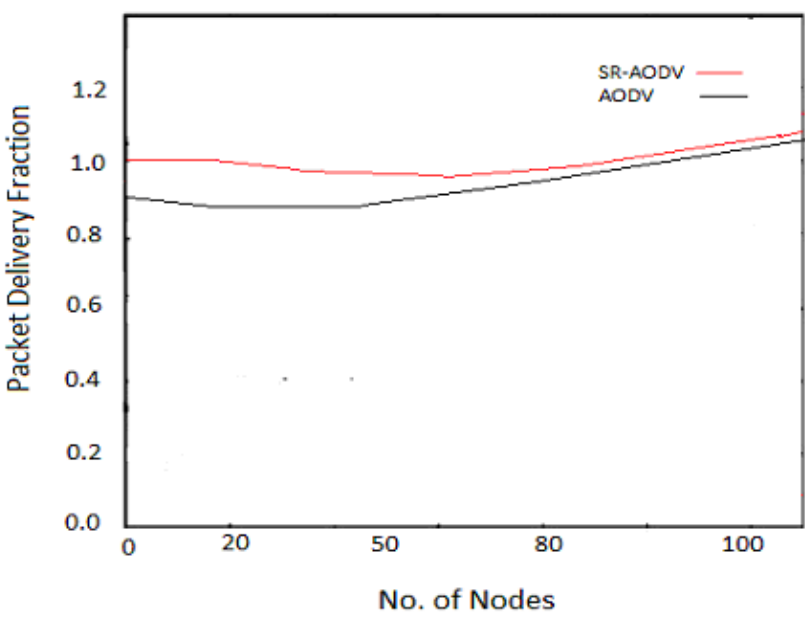

\section{Fig. 6.1: No. of Nodes versus Packet Delivery Fraction}

Fig. 6.1 shows at less node density, SR-AODV outperforms and it can adopt to the changes quickly; since it only maintain node reliability factor that is actively used. AODV deliver less data packet compare to SR-AODV because in rapid change topology AODV can adopt to the changes quickly since it only maintain one route i.e. actively used. Where as SR-AODV maintain both one route i.e. actively used and node reliability factor.

The quality of service provided by the protocols can be compared with the average delay. Fig.6.2 and Fig. 6.3 present the comparisons of the average end to end delay for SRAODV and AODV for 100 and 1000 nodes. The average end to end delay decreases with reduced mobility SR-AODV and AODV protocol. AODV shows largest delays in situations with high mobility due to its single path nature. SR-AODV, on the other hand, shows low delays in both cases.

Fig. 6.2: Average ETE Delay with 100 nodes

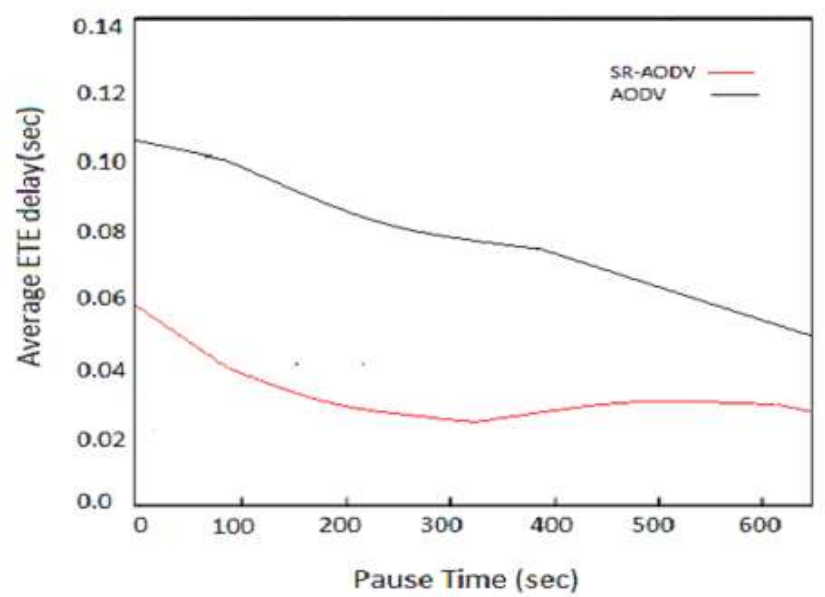

Fig. 6.3: Average ETE Delay with 1000 nodes

\section{CONCLUSION}

We have presented a modification of AODV routing protocol which is based on the path stability. We modified the AODV protocol and introduced the SR-AODV protocol. According to the simulation results, modified AODV showed a better performance in comparison to conventional AODV in packet delivery fractions in mobility of nodes. The connection setup delay is less in modified AODV in comparison to conventional AODV. So the average end to end delay is less in modified AODV in comparison to conventional AODV. The simulation results show that our new proposed protocol outperforms the conventional point text, as you see here. Please use sans-serif or non-proportional fonts only for special purposes, such as distinguishing source code text. If Times Roman is not available, try the font named Computer Modern Roman. On a Macintosh, use the font named Times. Right margins should be justified, not ragged.

\section{REFERENCES}

[1] Basagni S., Conti M., Giordano S., Stojmenovic I., "Mobile Ad Hoc Networking," IEEE Press, Wiley Interscience, 2004.

[2] Corson S., Macker J., "Mobile Ad Hoc Networking (MANET): Routing protocol performance issues and evaluation considerations," IETF RFC 2501, 1999. 
[3] Perkins C. E., Bhagwat P., "Highly dynamic destinationsequenced distance-vector routing (DSDV) for mobile computers," Computer Communications Review (1994), pp. 234-244.

[4] Agrawal C.P., Tiwari M. K., Vyas O.P., "Evaluation of AODV Protocol for Varying Mobility Models of MANET for Ubiquitous Computing", Third IEEE International Conference on Convergence and Hybrid Information Technology,2008, pp.769-774.

[5] Jianwu Z., Jingyuan Z., Qi Z., "MANET Routing Protocol for improving Routing Discovery Based on AODV", Intl. Conf. on Networks Security, Wireless Communications and Trusted Computing, 2009, pp.197200 .

[6] Wang H.P., Cui Lin, "An Enhanced AODV For Mobile Ad Hoc Network", Proceedings of the Seventh International Conference on Machine Learning and Cybernetics, Kunming, 12-15 July 2008, pp.1135-1140.
[7] Qiang Z., Hongbo Z., “An Optimized AODV protocol in mobile ad hoc network", 2008, pp.1-4.

[8] Marina M.K., Das S. R., "On-Demand multipath distance vector routing in ad hoc networks", Ninth Intl. Conf. on Network Protocols, 11-14 Nov. 2001, pp.14-23.

[9] Lu Q., Du L., Zuo Z., Xiao X., "Improved Multi-path AODV Protocols for Real-time Video Transport over Mobile Ad Hoc Networks", IEEE Pacific-Asia Workshop on Computational Intelligence and Industrial Application, 2008.

[10] McCanne S. and Floyd S. ns2 network simulator 2. [Online]. Available at: http://www.isi.edu/nsnam/ns, December 2003.

[11] Chung Jae and Claypool Mark, "NS by example 